\title{
VGLUT1 Binding to Endophilin or Intersectin1 and Dynamin Phosphorylation in a Diurnal Context
}

\author{
Karin Richter, ${ }^{\mathrm{a}}$ Isabelle Schmutz, ${ }^{\mathrm{b} \dagger}$ Mahesh Darna, ${ }^{\mathrm{a}}{ }^{\mathrm{j}}$ Johannes-F Zander, ${ }^{\mathrm{a}}$ Rohit Chavan, ${ }^{\mathrm{b}}$ Urs Albrecht ${ }^{\mathrm{b}}$ and \\ Gudrun Ahnert-Hilger ${ }^{\text {a* }}$ \\ a Institute for Integrative Neuroanatomy, AG Functional Cell Biology, Charité-University Medicine Berlin, Germany \\ ${ }^{\mathrm{b}}$ Department of Biology, Unit of Biochemistry, University of Fribourg, Switzerland
}

\begin{abstract}
Glutamate is concentrated into synaptic vesicles (SV) by the vesicular glutamate transporters (VGLUT) 1 and 2. VGLUTs also harbor a $\mathrm{Na}+/ \mathrm{Pi}$-transport activity when residing at the plasma membrane. Here we aimed to identify whether the diurnal switches of VGLUT1 parallels interactions with or modification of endocytic proteins. VGLUT1 and dynamin bind to SH3 domains of either endophilin (Enph) or intersectin 1 (ITSN1) harboring one or five SH3 domains A-E, respectively. We followed diurnal variations by pull down experiments using SH3 fusion protein and brains from mice entrained in a strict 24-h light-dark cycle (12-h light Zeitgeber (ZT) 0, 6; 12-h dark ZT 12 and 18). In pull downs with EnphSH3 interaction with VGLUT1 is high during the resting light and reduced during the active dark period while dynamin binding does not vary. This diurnal light/dark pattern depends on a functional period 2 gene and changes when animals are kept in complete darkness. Pull downs using ITSN1SH3 A reveal diurnally varying binding of VGLUT1 with slightly reduced VGLUT1/dynamin ratios at the beginning of the light (ZT 0) or the dark (ZT 12) period. Phosphorylation increases binding of VGLUT1 but not of dynamin to EnphSH3. In contrast binding of dynamin to ITSN1SH3 A decreases under phosphorylating conditions with no changes in VGLUT1 binding. Phosphorylation of dynamin at Ser 774 is high at ZT 6 and ZT 18 when more VGLUT1 is at the plasma membrane but low at ZT 0 and ZT 12 the diurnal peaks of VGLUT1 endocytosis. In conclusion the diurnally varying endocytosis of VGLUT1 involves differential interactions with the SH3 domains of Enph and ITSN1 and correlates with the de-phosphorylation of dynamin1. (c) 2017 IBRO. Published by Elsevier Ltd. All rights reserved.
\end{abstract}

Key words: vesicular glutamate transporter 1, circadian rhythmicity, endocytosis, endophilin, intersectin1, dynamin1 phosphorylation.

\section{INTRODUCTION}

Brain functions vary over the day, which is partly due to changes in the sequential change of light and darkness. The diurnal coordination of individual cellular clocks maintaining complex brain functions are orchestrated by a master clock located in the suprachiasmatic nucleus (SCN). At the molecular level, daily rhythms in different tissues, including brain, involve CLOCK and BMAL1 which induce transcription of Period (Per1, Per2 and Per3) and Cryptochrome (Cry1 and Cry2) genes. The

*Correspondence to: G. Ahnert-Hilger, AG Functional Cell Biology, Institute for Integrative Neuroanatomy, Charité-University Medicine Berlin, Philippstr. 12, 10115 Berlin, Germany.

E-mail address: gudrun.ahnert@charite.de (G. Ahnert-Hilger).

$\dagger$ Present address: Laboratory for Cell Biology and Genetics, the Rockefeller University, New York, USA.

* Present address: Department of Pharmaceutical Sciences, University of Kentucky, USA.

Abbreviations: ITSN1, intersectin; Enph, endophilin; VGLUT1, vesicular glutamate transporter; SV, synaptic vesicle; ZT, Zeitgeber; $\mathrm{CT}$, circadian time.
Per and Cry gene products in turn inhibit the activity of CLOCK and BMAL1 and thereby constitute a negative feedback loop (Albrecht and Eichele, 2003).

The daily changes of the environment require adaption of synaptic function in various neuronal systems including neurotransmission at individual synapses (Albrecht and Eichele, 2003; Tsanov and Manahan-Vaughan, 2007). Glutamate is the major excitatory neurotransmitter in the mammalian CNS. Following exocytosis glutamate is cleared from the extracellular space by plasma membrane glutamate transporters mainly located on astroglial cells and transported back to neurons involving the glutamine-glutamate cycle. Inside neurons, glutamate is concentrated into synaptic vesicles (SV) by three structurally related vesicular transporters (VGLUT). VGLUT1 and VGLUT2 are expressed in brain with partially overlapping patterns and cover the majority of glutamatergic signaling (Edwards, 2007; El Mestikawy et al., 2011). Several copies of either VGLUT1 or VGLUT2 are found on SV (Takamori et al., 2006). Although a single-VGLUT molecule is sufficient to fill an 
SV if time permits (Daniels et al., 2006) the copy number of VGLUT per SV affects synaptic efficiency (Moechars et al., 2006).

When analyzing whole-brain SVs prepared from mice sacrificed at different times of the day, the amounts of VGLUT1 and VGLUT2 vary while other SV proteins including the vesicular GABA transporter remained unaltered. The reduction in VGLUT on SV correlated with a reduced uptake of ${ }^{3} \mathrm{H}$ glutamate (Yelamanchili et al., 2006), (Darna et al., 2009). The observed pattern differed when mice were kept in complete darkness or when using mice expressing non-functional PER2 (Per2 ${ }^{\text {Brdm1 }}$ mice) (Yelamanchili et al., 2006). Pronase digestion of mouse synaptosomes, (the starting fraction of the SV preparation), reflects the diurnal variations of VGLUT1 amounts on SVs (Darna et al., 2009). These data confirm a diurnal sorting of VGLUT1 to and from the plasma membrane, which is not shared by other SV proteins and support a diurnal control of stored glutamate at the presynaptic level. Initially VGLUT1 and VGLUT2 have been identified as sodium/phosphate transporters of the plasma membrane (Ni et al., 1994) (Aihara et al., 2000). Residing transiently at the plasma membrane VGLUT1 may restore cytoplasmic phosphate pools.

Endocytosis of VGLUT1 in a diurnal context is supposedly mediated by a unique reversible sequestration into the endocytic protein network that scaffolds the periactive zone surrounding sites of SV fusion. Generally, endocytosis of SV proteins involves clathrin and the adaptor protein AP2. Furthermore, endophilin (Enph) and intersectin (ITSN) 1 can initiate the formation of scaffolding platforms and organize the assembly of huge protein complexes thereby gradually promoting endocytosis. Enph by its single $\mathrm{SH} 3$ domain binds to the second proline-rich motif of VGLUT1 and to dynamin (De Gois et al., 2006; Vinatier et al., 2006; Voglmaier and Edwards, 2007). ITSN1 consists of five $\mathrm{SH} 3$ domains $(\mathrm{A}-\mathrm{E})$ and has been shown to interact with endocytic proteins including dynamin, synaptojanin, AP2, and Enph (Pechstein et al., 2010; Pechstein et al., 2015). So far, the molecular details of the specific exoendocytic cycling of VGLUT1 reflecting its variations on SVs in diurnal context (Yelamanchili et al., 2006; Darna et al., 2009) are still obscure. Here we describe that the VGLUT1 interaction with either Enph or ITSN1SH3 A changes in a time-of-day-dependent manner. Phosphorylation of dynamin decreases its interaction with ITSN1SH3 $A$ and negatively correlates with the diurnal VGLUT1 endocytosis.

\section{EXPERIMENTAL PROCEDURES}

\section{Materials}

The following antibodies were used:

Rabbit anti-endophilin 1 (159 002) Synaptic Systems, Göttingen, Germany

Rabbit anti-VGLUT2 (135 402) Synaptic Systems, Göttingen, Germany

Guinea pig anti-VGLUT2 (135 404) Synaptic Systems, Göttingen, Germany
Rabbit anti-VGLUT1 (135 302) Synaptic Systems, Göttingen, Germany

Guinea pig anti-VGLUT1 (135 304) Synaptic Systems, Göttingen, Germany

Mouse anti-VGLUT1 (135311) Synaptic Systems, Göttingen, Germany

Rabbit anti-dynamin (115002) Synaptic Systems Göttingen, Germany

Rabbit anti-dynamin Ser 774 (148899) BioRad Laboratories, Berkeley, California, USA

Rabbit anti-gst (ab9085), Abcam, Cambridge, UK

Mouse anti-synaptojanin 1 (sc-32770) Santa Cruz, California, USA

Mouse anti-alpha adaptin (AP2- $\alpha$ ) (MA3-061) Dianova, Hamburg, Germany

Rabbit anti-intersectin (ab118262), Abcam, Cambridge, UK

Mouse anti-intersectin/ESE-1 (611574), BD Transduction Laboratories, New Jersey, USA

Mouse anti-synaptophysin 1 (101011) Synaptic Systems, Göttingen, Germany

\section{Mice}

Per2 ${ }^{\text {Brdm1 }}$ mice used in this study were characterized previously (Zheng et al., 1999). The wild-type and Per2 ${ }^{\text {Brdm1 }}$ animals used represent littermates derived from intercrosses between heterozygous Per2 ${ }^{\text {Brdm1 }}$ mice on a 129SvEvBrd/C57/BL/6-Tyrc-Brd background. Mice were kept under either an uninterrupted 12-h light/12-h dark or an uninterrupted dark/dark cycle for at least 7 or 3 days, respectively, before the start of the experiment (Albrecht and Foster, 2002). Experiments were performed in accordance with the guidelines of the Swiss animal laws (TSchG, SR455) and the declaration of Helsinki. The protocol was approved by the state veterinarian of the Canton of Fribourg.

\section{GST-fusion proteins and peptides}

VGLUT1 c-terminal part was amplified from a mouse brain library using the following primer pairs:

\section{Fwd: GGG GAA TTC TCG GGA GAG AAG CAG} Rev: GGG CTC GAG TCA GTA GTC CCG GAC

The respective cDNA was expressed in E. coli BL21 cells using EcoRI-Xhol fragments of the pGEX4T1 (GE Healthcare) vector to yield GST fusion proteins. DNA sequencing (SMB Services in Molecular Biology, Berlin, Germany) verified sequences.

GST (glutathione-S-transferase) fusion proteins of the $\mathrm{SH} 3$ domain of endophilin (EA1-SH3, referred to as EnphSH3) and a mutant containing an exchange of glycine to arginine at position 337 and of proline to leucine at position 340 (referred to as EnphSH3-GPRL) were kindly provided by V. Haucke FMP Berlin Buch Germany. GST-fusion proteins of the SH3 domains of human intersectin1 (Pechstein et al., 2010), ITSN1SH3 A-E, and ITSN1SH3 A, were kindly provided by Arndt Pechstein, (FMP Berlin Buch Germany) and ITSN1SH3 
B, by Christian Freund (Inst. of Chemistry and Biochemistry FU, Berlin, Germany).

A dynamin peptide covering the amino acids 782-799 (QRRAPAVPPARPGSRGPA-NH) (Ringstad et al., 2001) was kindly provided by Christian Freund Inst. of Chemistry and Biochemistry, FU Berlin.

An overview of GST fusion proteins is given in Table 1.

\section{Expression of GST fusion proteins}

Expression of GST fusion proteins was induced at $\mathrm{OD}_{600}$ $0.5-0.8$ by $0.5 \mathrm{mM} \mathrm{IPTG}$ at $30{ }^{\circ} \mathrm{C}$ for $4 \mathrm{~h}$ in $2 \mathrm{YT}$ medium (16 g/l Tryptone, $10 \mathrm{~g} / \mathrm{l}$ Yeast Extract, $5 \mathrm{~g} / \mathrm{l} \mathrm{NaCl}$ ) containing $100 \mathrm{mg} / \mathrm{ml}$ Ampicillin. Bacterial cells were harvested by centrifugation at $3000 \mathrm{~g}$ and suspended in PBS $(20 \mathrm{ml} / 250 \mathrm{ml}$ culture). For lyses, $500 \mu \mathrm{g}$ of lysozyme was added together with RNAse and DNAse $(10 \mu \mathrm{l} / 20 \mathrm{ml})$ and protease inhibitors (1:1000, Sigma) and PMSF (1mM, Sigma). After incubation on ice for 15 min Triton X-100 (Roche) was added in between two sonification pulses for $90 \mathrm{~s}(50-60 \mathrm{kHz}, 60 \%$ power, $50 \%$ duty cycle). Lysed cells were spun at maximum $30,000 \mathrm{~g}$ force and filtered supernatants were incubated with 1-2 $\mathrm{ml}$ Glutathione Sepharose (GE Healthcare) for $1 \mathrm{~h}$ at $4{ }^{\circ} \mathrm{C}$ on rotating wheel. Beads were washed three times with PBS before being used for pull down assay.

\section{Circadian studies}

Mice were sacrificed at the given time points either in the 12-h light/12-h dark cycle (Zeitgeber time (ZT)) or the dark/dark cycle (circadian time (CT)). Under appropriate conditions, wild-type and Per2 $2^{\text {Brdm1 }}$ mutants were analyzed in parallel. Protein determination was performed by the BCA method, and equal amounts of protein were loaded for SDS-PAGE.

For diurnal analyses of protein interactions animals were sacrificed at the indicated time points, brains were quickly removed and frozen in liquid nitrogen until further processing. From each sample (animal) three different dilutions of the pull down solution were analyzed and first normalized to GST (VGLUT1 and dynamin separately) before the ratio VGLUT1 to dynamin was calculated. Signals in the non-saturated range were taken for quantification.

For each set of experiments, brain extracts were run in parallel, and proteins were transferred to nitrocellulose and further processed for immunodetection using the $E C L$ detection system. ECL-processed films were scanned by video imaging, and protein bands were densitometrically quantified using the SCAN PACK 3.0 program. It was ensured by loading different amounts for each sample that the signals were in the linear range of the ECL detection system (Brunk et al., 2006; Zander et al., 2010). Quantification was performed as described (Brunk et al., 2006; Zander et al., 2010) along with statistical analysis using paired Student's $t$ test.

\section{Triton extraction, pull down assay and immunoprecipitation}

Post nuclear fractions or synaptosomes of adult mice brain $(5 \mathrm{mg} / \mathrm{mL})$ were extracted for $1 \mathrm{~h}$ at $4{ }^{\circ} \mathrm{C}$ on a rotor shaker in HMK buffer (20 mM Hepes buffer ( $\mathrm{pH} 7.4), 50$ $\mathrm{mM} \mathrm{KCl}, 2 \mathrm{mM} \mathrm{MgCl}$ ) containing 1\% (v/v) Triton X-100. Samples were diluted to 1 or $2 \mathrm{mg} / \mathrm{mL}$ and supplemented with protease and phosphatase inhibitors (cocktail set V, Calbiochem) and centrifuged at $2700 \mathrm{~g}$ for $5 \mathrm{~min}$ to separate extractable from non-extractable fractions (Becher et al., 1999). Extracts were used for pull down or immunoprecipitation.

For pull down experiments 100 or $50 \mu \mathrm{g}$ GST-fusion proteins were coupled to Glutathione Sepharose fast flow beads (GE Healthcare) via incubation for $1 \mathrm{~h}$ at 4 ${ }^{\circ} \mathrm{C}$. The protein-bead complex was incubated with mouse brain extract ( $2 \mathrm{mg}$ protein) in a total volume of 1 $\mathrm{ml}$ for $1 \mathrm{~h}$ at $4^{\circ} \mathrm{C}$ on a rotating wheel. Supernatants were removed and diluted with sample buffer. After two washing steps with HMK buffer containing $1 \%$ Triton $\mathrm{X}$ -

Table 1. Overview of GST fusion protein and antibodies for immunoprecipitation.

\begin{tabular}{|c|c|c|c|c|}
\hline \multicolumn{5}{|l|}{ Pull down experiments } \\
\hline Fusion protein & Protein in PD & & & \\
\hline \multirow[t]{3}{*}{ EnphSH3 } & VGLUT1 & Dynamin & ITSN1 & \\
\hline & ATP increases binding & & & \\
\hline & Dynamin peptide interferes & Dynamin peptide interferes & & \\
\hline EnphSH3-GPRL & VGLUT1 & & & \\
\hline cVGLUT1 & & & & Enph \\
\hline \multirow[t]{3}{*}{ ITSN1SH3 A } & VGLUT1 & Dynamin, & & \\
\hline & & ATP decreases binding & & \\
\hline & Dynamin peptide interferes & Dynamin peptide interferes & & \\
\hline ITSN1SH3 B & VGLUT1 & & & Enph \\
\hline ITSN1SH3 A-E & VGLUT1 & Dynamin & & Enph \\
\hline \multicolumn{5}{|l|}{ Immunoprecipitations } \\
\hline Antibody against & Protein in IP & & & \\
\hline Enph & VGLUT1 & & & Enph \\
\hline VGLUT1 & VGLUT1 & & ITSN1 & \\
\hline
\end{tabular}

The impact of putative phosphorylation (difference in binding between presences of ATP or calf intestinal phosphatase) is indicated (see Figs. 1 and 3). Interference with a dynamin peptide is indicated (see Fig. 1). Enph is not seen in IP with VGLUT1 since the antibody is directed against the c-terminus. 
100, pull down samples were washed once in HMK buffer and eluted with $100 \mu \mathrm{l}$ sample buffer. Samples were analyzed by SDS/PAGE and immunoblotting.

Immunoprecipitations were performed as given previously (Becher et al., 1999) using $200 \mu \mathrm{l}$ of extract and $2-5 \mu \mathrm{g}$ of the indicated antibody coupled to $50 \mu \mathrm{l}$ Dynabeads Protein G or A (Invitrogen) according to the manual (Dynabeads, Cat. no. 100.03D).

\section{Western blotting}

Protein samples solubilized in $1 \times$ SDS (sodium dodecyl sulfate) sample buffer (50 mM Tris, $100 \mathrm{mM}$ DTT, 2\% SDS, $0.1 \%$ bromophenol blue, $10 \%$ glycerin) were separated by SDS-PAGE and electro-transferred onto nitrocellulose Hybond C (Amersham, GE Healthcare). Immunosignals were visualized by either anti-mouse, anti-rabbit or anti-goat antibodies coupled to horseradish peroxidase (Vector Laboratories 94010). To avoid interference between IgG heavy chain and the VGLUT1 signal, light chain-specific secondary antibodies were applied (Dianova). Stained bands were densitometrically analyzed using the Lablmage 1D 2006 (Kapelan BioImaging Solutions) (Zander et al., 2010).

\section{RESULTS}

\section{Diurnal variations of the Enph/VGLUT1interaction}

Using a GST fusion protein of the SH3 domain of EnphA1 (EnphSH3) we confirm the described interaction with VGLUT1 (De Gois et al., 2006; Voglmaier et al., 2006). VGLUT1 in addition binds to modified EnphSH3 domain (SH3-GPRL) in which the conserved amino acids relevant for binding to $\mathrm{SH} 3$ domains have been exchanged (Grabs et al., 1997) while dynamin does not bind (Fig. 1A). VGLUT2 does not bind at all (Voglmaier et al., 2006). The impact of putative phosphorylation was analyzed by adding either calf intestinal phosphatase (CIP) to deplete ATP or ATP to brain lysate before pull down with EnphSH3. Addition of ATP increases the VGLUT1 binding while the interaction with dynamin remains unchanged (Fig. 1A). When using a C-terminal GST-fusion protein of VGLUT1 for pull down phosphorylating condition has no impact on Enph binding (Fig. 1B, Table 1) supporting recently described data for phosphorylation of VGLUT1 at serine 519 and 522 (Santos et al., 2014). The dynamin peptide (aa782-799) diminishes the interaction between EnphSH3 and VGLUT1 (Fig. 1C) indicating overlapping binding sites for dynamin and VGLUT1.

Next we analyzed the Enph/VGLUT1 interaction in context of a day-night cycle applying the GST-EnphSH3 fusion protein to whole-brain extracts of wild-type and Per2 ${ }^{\text {Brdm1 }}$ mice, entrained in a 12-h light/12-h dark (LD) cycle. The precipitated proteins were detected by Western blot analysis and the obtained bands quantified. Under the LD conditions the amounts of VGLUT1 on SV are high at the beginning of the light (ZT0) or dark (ZT12) period and low in the middle of the day (ZT6) or night (ZT12) due to a diurnally controlled transient stay of some VGLUT1 copies at the plasma membrane (Yelamanchili et al., 2006; Darna et al.,

\section{A PD GST-EnphSH3}
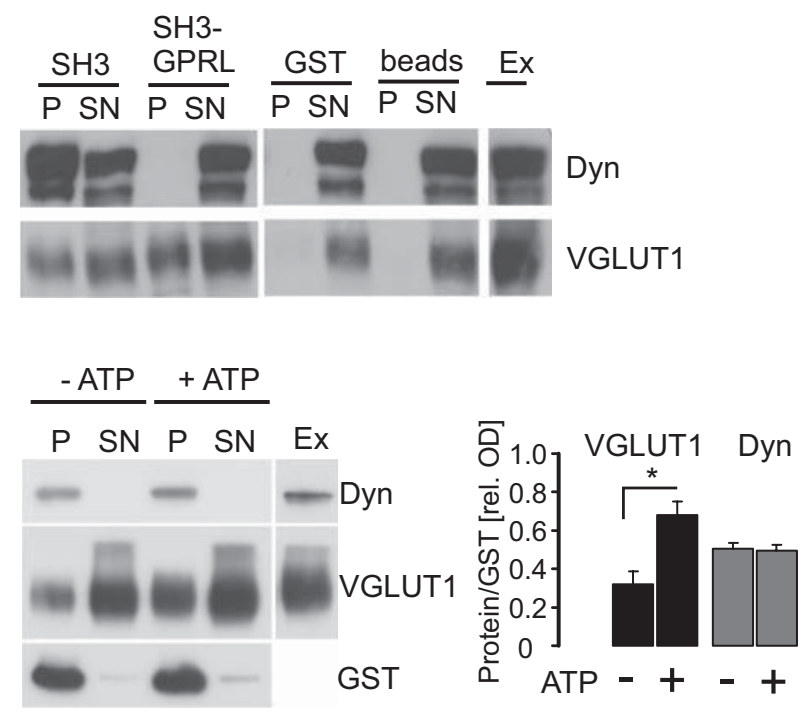

B PD GST-cVGLUT1
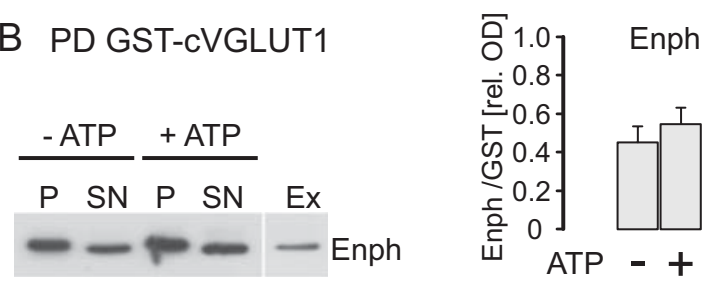

C PD GST-EnphSH3

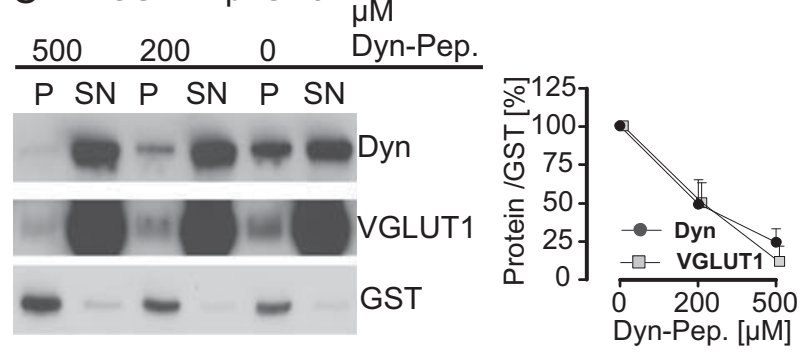

Fig. 1. Pull down with endophilin (Enph) SH3 GST fusion proteins (A) Pull down (PD) from whole-mouse brain extract using EnphSH3 shows binding of VGLUT1 $(60 \mathrm{kDa})$ and dynamin $(110 \mathrm{kDa})$. The interaction with dynamin is, not seen when the EnphSH3 domain is varied (GPRL). In contrast, VGLUT1 binding to EnphSH3 is also seen when using the mutated EnphSH3-GPRL fusion protein for PD. Extract (Ex) represents $10 \%$ of the starting material. Phosphorylation (addition of either ATP or calf intestinal phosphatase (-ATP) to pull down) increases the EnphSH3/VGLUT1 interaction with no effect on the EnphSH3/dynamin interaction. (B) When precipitating endophilin (Enph, $40 \mathrm{kDa}$ ) using C-terminal fusion protein (GST-cVGLUT1) ATP has no effect on the interaction with Enph. (C) The dynamin peptide (QRRAPAVPPARPGSRGPA-NH) diminishes the binding of dynamin (Dyn) or VGLUT1 to SH3 endophilin. $200 \mu \mathrm{M}$ peptide results in a $50 \%$ inhibition while $500 \mu \mathrm{M}$ peptide almost abolishes binding of dynamin or VGLUT1 to EnphSH3. Values represent relative optical densities (OD) of the given protein. Quantification using GST (fusion protein 26 $\mathrm{kDa}$ ) as reference represents the mean of three different experiments \pm S.D., using Student's $T$-test for determination of significance. 
2009). The Enph/VGLUT1 interactions are high before and during the daily light phase (ZTO and ZT6) but decrease at the beginning of the night, being still low at midnight (Fig. 2A upper left) not completely reflecting the endocytic pattern of VGLUT1. In the absence of a functional Per2 gene (Per2 $2^{B r d m 1}$ mice), this pattern is lost (Fig. 2A upper right). The pattern shifts when animals are kept in complete darkness (24-h dark DD, circadian time) with a peak of the Enph/VGLUT1 interaction at CT12 (Fig. 2B upper left). Under DD conditions the high VGLUT1/ EnphSH3 interaction correlates with the high amounts of VGLUT1 seen at CT12 (Yelamanchili et al., 2006). Again, there are no changes in the Enph/VGLUT1 interaction when analyzing Per2 ${ }^{\text {Brdm1 } 1}$ mice (Fig. 2B upper right). The Enph/dynamin interaction is not regulated diurnally and serves as an internal control. The overall amounts of dynamin and VGLUT1 do not vary under LD or DD as analyzed in the extracts used for pull down with synaptophysin (Syp) as a reference (Fig. 2A, B extract).

\section{Diurnal variations in VGLUT1/ ITSN1 interactions}

Intersectin1 (ITSN1) contains five $\mathrm{SH} 3$ domains which specifically interact with proline-rich domains (PRD) of a variety of proteins (Evergren et al., 2007; Pechstein et al., 2010). Using GST fusion proteins from ITSN1SH3 A-E we found a strong interaction with VGLUT1 (Fig. 3A), besides the known binding to AP2 and dynamin. ITSN1SH3 A alone also strongly interacts with VGLUT1 and dynamin. Enph only binds to ITSN1SH3 B but not to ITSN1SH3 A confirming recent observations ((Pechstein et al., 2015) Fig. 3). The binding of VGLUT1 to ITSN1SH3 B may be indirect and mediated by Enph (see Fig. 1) while dynamin is not precipitated by ITSN1SH3 B (Fig. 3A). AP2 is only seen when all five $\mathrm{SH} 3$ domains of ITSN1 were used for pull down in agreement with the AP2 binding to the linker region of ITSN1SH3 A/B domains (Pechstein et al., 2010). Neither VGLUT2 nor Syp is precipitated. The interaction with ITSN1 is confirmed by immunoprecipitation using an antibody against VGLUT1 (Fig. 1B). No interaction with

B dark-dark
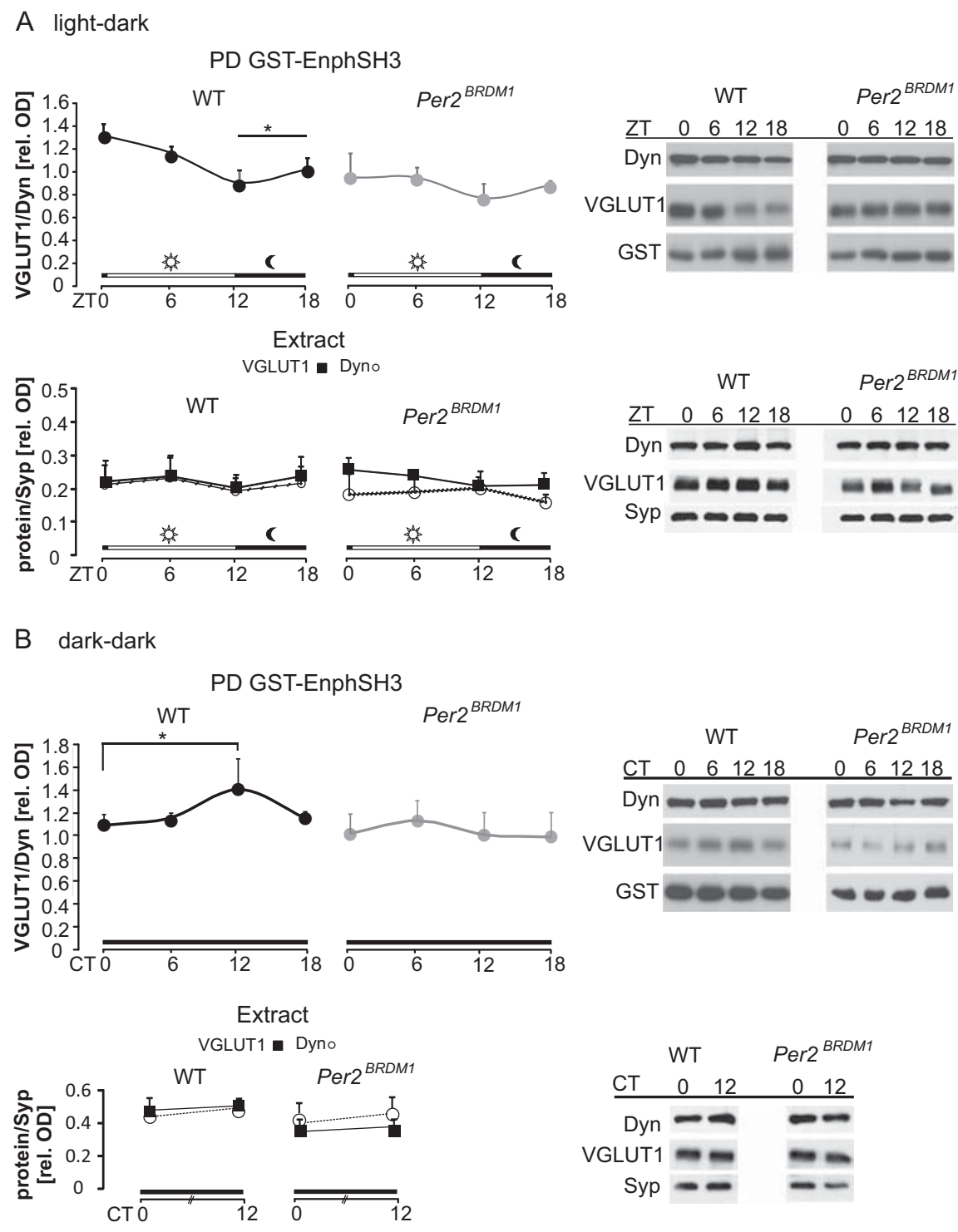

Fig. 2. Diurnal variations of the Enph/VGLUT1 interaction. PD assays with EnphSH3 fusion protein were performed using brains from mice kept under light/dark (LD cycle, ZT) or dark/dark (DD cycle, CT) conditions. The time points are ZTO representing dawn at $6.00 \mathrm{am}$, before the light is switched on, ZT6 at noon in the middle of the light phase ZT12 dusk at $6.00 \mathrm{pm}$ (before the light is switched off) and ZT18 in the middle of the night. CT0-18 refers to the corresponding time points, respectively, under constant darkness. A: Binding of VGLUT1 to Enph varies in context of a lightdark cycle (indicated by the white-black line) while dynamin binding to Enph remains unchanged. Binding is high at ZT0 and ZT6 compared to ZT12 and ZT 18. Mice lacking a functional PER2 protein $\left(P e r^{2 B R D M 1}\right)$ exhibit no variations in VGLUT1 or dynamin binding to Enph. Amounts of VGLUT1 and dynamin given in the corresponding extracts (the starting fraction for PD) analyzed in parallel with synaptophysin (Syp) as reference do not vary in a diurnal context. The ratio between VGLUT1 and dynamin bound to EnphSH3 is given. Values represent the mean ( \pm S.D.) of three $(Z T 6,18)$ or five $(Z T 0,12)$ animals per individual data point and sacrificed at the indicated time points. An asterisk indicates significance (ZT0-ZT12: 0.0004; ZT0-ZT18: 0.02; Student's T-test). (B) When mice are kept in complete darkness (dark-dark, indicated by the black line) the pattern reverses. Now binding of VGLUT1 is higher at CT12 compared to CTO. Again, Per $r^{2 B R D M 1}$ mice show no changes over all time points. Amounts of VGLUT1 and dynamin given in the corresponding extracts do not vary. The ratio between VGLUT1 and dynamin bound to EnphSH3 is calculated as relative optical densities (OD). Values represent the mean \pm S.D. of four (CT6, 18) or six animals $(\mathrm{CTO}, 12)$ per individual data point, sacrificed at the indicated time points. An asterisk indicates significance (CT0-CT12: 0.05; Student's T-test).

ITSN1 could be detected when using the C-terminal part of VGLUT1 for precipitation (Voglmaier et al., 2006), indicating that other parts or modifications of VGLUT1 are 
required for the interaction with ITSN1. Phosphorylation in the VGLUT1 C-terminus at Ser519 and Ser522 appears to increase binding of VGLUT1 to AP2 (Santos et al., 2014). We therefore studied the impact of putative phosphorylation by adding either calf intestinal phosphatase (CIP) or ATP to brain lysate before precipitating with ITSN1SH3 domains. Phosphorylation has no impact on the interaction of ITSN1SH3 A with VGLUT1 but decreases the binding to dynamin (Fig. 1C, Table 1). Since both dynamin and VGLUT1 bind to ITSN1SH3 A

\section{A PD GST-ITSN1SH3}

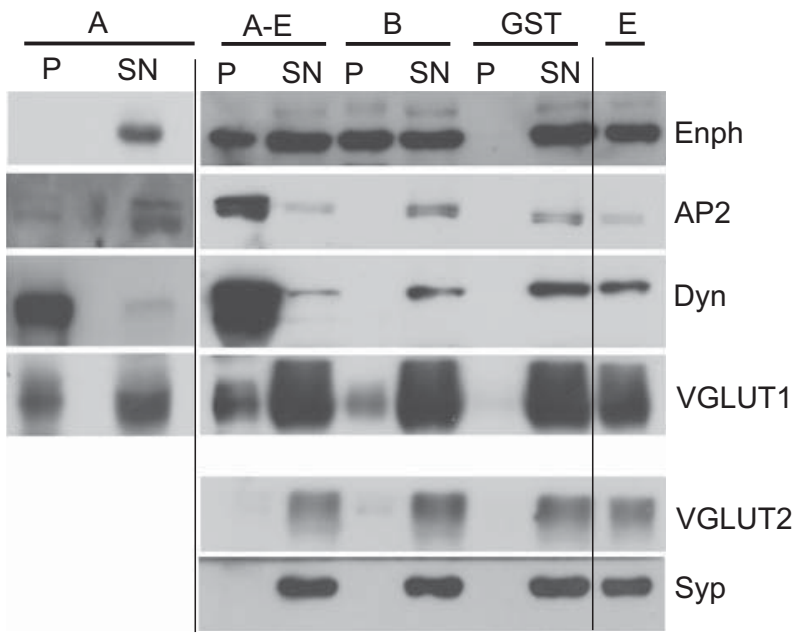

B IP with $A b$

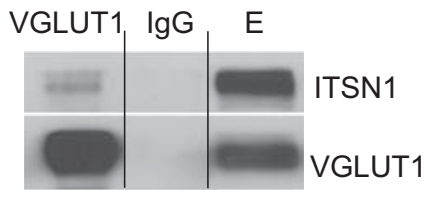

C PD GST-ITSN1SH3 A
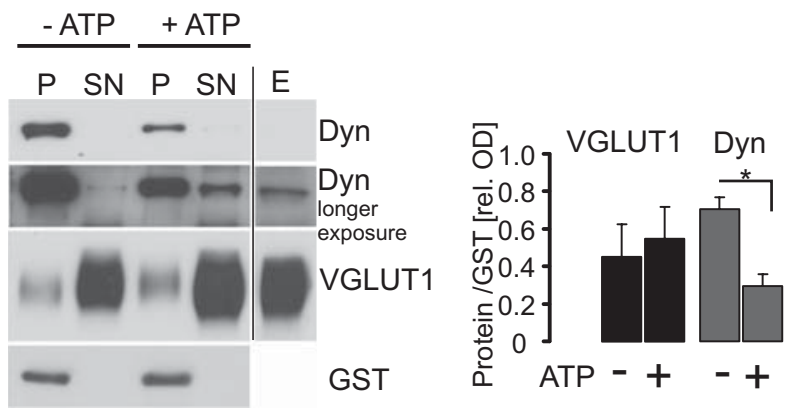

D PD GST-ITSN1SH3 A

$\begin{array}{lll}\mu M \\ 0 \quad 500200 & \text { Dyn-Pep. }\end{array}$

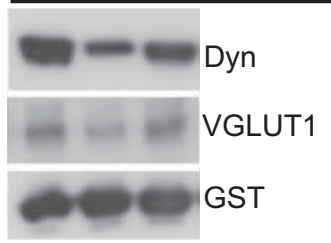

we analyzed a putative competition using the dynamin peptide covering aa 782-799 (Ringstad et al., 2001). As can be seen the peptide interferes with VGLUT1 binding to ITSN1SH3 A (Fig. 3D) suggesting that VGLUT1 and dynamin compete for a common binding site in the ITSN1SH3 A domain.

To study the ITSN1 interaction with VGLUT1 in a diurnal context we focussed on ITSN1SH3 A-E and ITSN1SH3 A using mice entrained in a LD cycle. Binding of VGLUT1 to ITSN1SH3 A-E only slightly varies over all time points (Fig. 4A). When using ITSN1SH3 A for precipitation the VGLUT1 binding is low at the beginning of the light phase (ZTO immediately before the light is switched on) and slightly increases at ZT6 (noon) while during the rest of the day (ZT 12 and ZT 18) no further changes occur (Fig. 4B). Dynamin binding to both ITSN1SH3 constructs does not vary over the $4 \mathrm{ZT}$ time points (Fig. 4A, B). Thus there are moderate diurnal variations of the ITSN1SH3 A interaction with VGLUT1 that however do not mimic the recently discovered diurnal changes of the amounts of VGLUT1 on SV (Yelamanchili et al., 2006).

\section{Diurnal variations in the phosphorylation of dynamin}

As seen above the dynamin aa 782-799 competes with VGLUT1 for binding to ITSN1SH3 A suggesting a common binding site for VGLUT1 and dynamin. In addition, the binding of dynamin to ITSN1SH3 $A$ is enhanced when dynamin is dephosphorylated (see Fig. 3C) (Pechstein et al., 2015). Dynamin1 dephosphorylation at Ser 774 accelerates endocytosis (Armbruster et al., 2013; Kohansal-Nodehi et al., 2016). Since slight phosphorylation-dependent variation in the binding to ITSN1SH3 A may have escaped detection when regarding the overall dynamin binding we analyzed the amounts of phosphorylated dynamin versus dynamin in brain extracts from mice killed at the given $\mathrm{ZT}$ time points. As can be seen in Fig. 5 phosphorylation of dynamin detected by an antibody specifically recognizing

Fig. 3. Pull down with Intersectin1 (ITSN1) $\mathrm{SH} 3$ fusion proteins Mouse brain extracts were subjected to pull downs (PD) using the indicated GST-fusion proteins of intersectin SH3 domains, GSTITSN1 A-E (containing al five SH3 domains) and GST-ITSN1SH3 A or GST-ITSN1SH3 B, representing only the first $(A)$ or the second $(B)$ $\mathrm{SH} 3$ domain. (A) Enph (40 kDa), adaptor protein 2 (AP2, $100 \mathrm{kDa})$ ), dynamin (Dyn, $110 \mathrm{kDa}$ ) and VGLUT1 $(60 \mathrm{kDa})$ are found in pull downs with ITSN1SH3 A-E, which however lack VGLUT2 (65 kDa) and synaptophysin (Syp, $38 \mathrm{kDa})$ ) serving as negative controls. No attached proteins are seen in the GST pull down. Extract (Ex) represents $10 \%$ of the starting material. ITSN1SH3 B only binds Enph and VGLUT1, while the ITSN1SH3 A domain binds VGLUT1 and dynamin. (B) Immunoprecipitation using anti-VGLUT1 confirmed the interaction with intersectin. (C) Phosphorylation (addition of ATP or calf intestinal phosphatase (-ATP) to pull down) decreases dynamin binding to ITSN1SH3 A compared to de-phosphorylation (application of calf intestinal phosphatase). The interaction with VGLUT1 analyzed in parallel is unaffected. Values given as relative optical density (OD) using GST as reference, represent the mean of three independent experiments \pm S.D. ${ }^{*}$ denotes significance. (D) The dynamin peptide (QRRAPAVPPARPGSRGPA-NH) partially inhibits the interaction between the SH3 A domain of ITSN1 with either VGLUT1 or dynamin. 


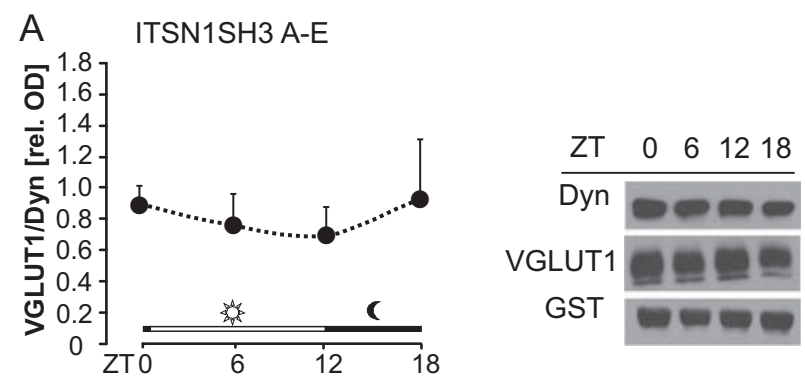

B ITSN1SH3 A

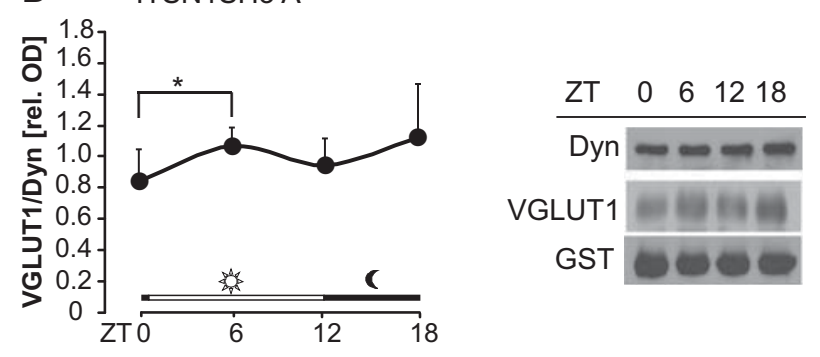

Fig. 4. ITSN1SH3/VGLUT1 interaction in day-night context. Pull downs $(\mathrm{PD})$ from brain extracts taken at the indicated time of the day using either ITSN1SH3 A-E (A) or ITSN1SH3 A (B) fusion proteins. Varying interactions of VGLUT1 and dynamin with ITSN1SH3 A occurs mainly between ZTO and ZT6 while the binding of VGLUT1 or dynamin to ITSN1SH3 A-E does not vary diurnally. The ratio between VGLUT1 and dynamin bound to ITSN1SH3 A-E (A) or to ITSN1SH3 A (B) is given. Values represent the mean \pm S.D. of eight animals per individual data point, sacrificed at the indicated time points. An asterisk indicates significance (ZTO-ZT6: 0.03; Student's $T$-test).

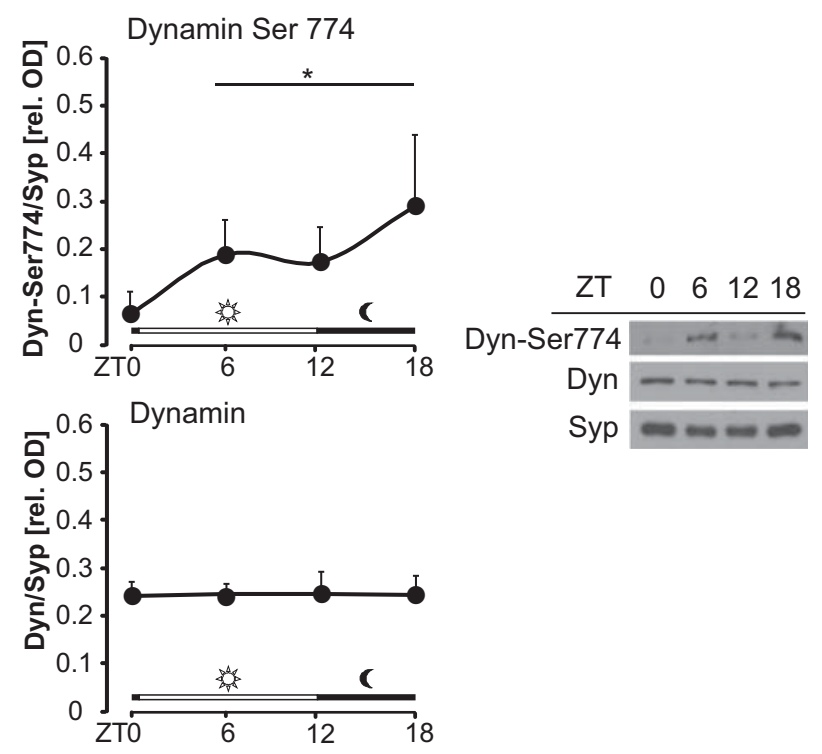

Fig. 5. Amounts of dynamin and phosphorylated dynamin in daynight context. Brain extracts taken at the indicated time of the day were analyzed for the content of dynamin and dynamin phosphorylated at Ser774. While the amounts of overall dynamin do not change the signal obtained with the antibody against DynSer774 is low at ZT0 higher in the middle of the day (ZT6) lower again at ZT12 (dawn) and high at midnight (ZT18). Synaptophysin (Syp) was determined in parallel and used as reference. Values represent the mean \pm S.D. of five animals per individual data point, sacrificed at the indicated time points. An asterisk indicates significance (ZT0-ZT6: 0.02; ZT0-ZT12: 0.04; ZT0-ZT18: 0.03; Student's T-test). dynamin phosphorylated at serine 774 (dyn Ser774) is lower at ZT0 and ZT12 compared to ZT6 and ZT18 while the overall dynamin content does not vary. Thus the amount of phosphorylated dynamin negatively reflects the amounts of VGLUT1 on SV being high at ZTO and ZT12 (Yelamanchili et al., 2006) indicating increased endocytic activity. On the other side, VGLUT1 may be kept at the plasma membrane when bound to ITSN1SH3 $A$ and when dynamin is phosphorylated as at least reflected by the higher VGLUT1/ITSN1SH3 A interaction at ZT6 (see Fig. 4B).

\section{DISCUSSION}

The variations of VGLUT1 on mouse brain SVs observed previously in a LD cycle (Yelamanchili et al., 2006; Darna et al., 2009) are rather specific for VGLUT1 and not shared by other SV proteins. The present study revealed first that VGLUT1 amounts on SVs negatively correlate with the phosphorylation of dynamin at Ser774 indicating that the availability of non-phosphorylated dynamin is crucial for VGLUT1 endocytosis. Second, endocytosis of VGLUT1 is mediated by interaction with ITSN1 besides the already known role of Enph. However, with both endocytic proteins the interaction pattern over a day-night cycle only partially reflects the varying amounts of VGLUT1 on SVs in a diurnal context. This may be due to additional roles Enph appears to play besides being a key protein of endocytosis. Regarding ITSN1 the five $\mathrm{SH} 3$ domains probably control different mutually exclusive steps during endocytosis (see also Table 1).

The effects of Enph in the diurnal endocytosis of VGLUT1 vary in context of a LD cycle and changes when animals are kept under dark-dark conditions (CT). The observed variations depend on a functional Per2 gene. Generally, Enph plays multiple roles depending on species as well as tissue and cell type (Bai et al., 2010; Milosevic et al., 2011). In rodent brain Enph is highly relevant for endocytosis by helping to uncoat SVs (Milosevic et al., 2011). Enph specifically interacts with the second proline-rich domain of VGLUT1 and this interaction has been described to mediate an alternative endocytic pathway involving AP180 (Voglmaier et al., 2006). However, in the Enph knockout mice no specific modulation of VGLUT1 endocytosis compared to the majority of other SV proteins could be observed (Milosevic et al., 2011). The Enph binding to ITSN1SH3 B may bridge segregated steps in retrieving VGLUT1 from the plasma membrane. Enph may promote endocytosis of VGLUT1 by guiding it to the other ITSN1SH3 domains including ITSN1SH3 B. Interestingly dynamin is absent in ITSN1SH3 B pull downs although Enph is bound. So it may be also feasible that Enph mediates VGLUT1 binding to ITSN1 via its SH3 B domain and thereby further promotes VGLUT1 endocytosis.

Enph increases release probability in its dimeric form. Its binding to VGLUT1 decreases the release probability of VGLUT1 containing SV and synaptic efficiency in glutamatergic synapses (Weston et al., 2011). The Enph/VGLUT1 interaction may be transient or longer lasting and is therefore not always discernible from Enph's 
effect on endocytosis (Weston et al., 2011). The Enph/ VGLUT1 interaction does not impair vesicular glutamate loading (Vinatier et al., 2006). In a day-night context, the high Enph/VGLUT1 interaction appears to reflect the reduced activity during day and its decrease the high activity during night. Thus the diurnal changes observed in the VGLUT1/Enph interaction represent an intermingling between variations in endocytosis and release probability. The modulation of the release probability mediated by VGLUT1 bound to Enph may provide a functional explanation for the extended vesicular synergy of VGLUT1 and VGLUT2 (Gronborg et al., 2010; Zander et al., 2010).

VGLUT1 binds to ITSN1. Which part of the VGLUT1 mediates the interaction is still unclear. VGLUT1 competes with dynamin for binding to ITSN1SH3 A. Phosphorylation decreases dynamin binding to ITSN1SH3 A. If phosphorylated dynamin is high at ZT6 and ZT18 the ITSN1SH3 A/VGLUT1 interaction may persist and keep the transporter at the plasma membrane reflected by lower amounts on SVs at these time points (Yelamanchili et al., 2006; Darna et al., 2009). Phosphorylation has no impact on VGLUT1 binding to ITSN1SH3 A. Phosphorylation enhances the interaction of VGLUT1 with Enph which may promote further steps for VGLUT1 endocytosis. These steps may involve a dissociation from ITSN1SH3 A thereby allowing nonphosphorylated dynamin to bind. Changes in the phosphorylation by adding or removing ATP does not affect binding of VGLUT1 to ITSN1SH3 A. Combined these observations suggest that ITSN1SH3 A either interacts with one of the cytosolic loops or with VGLUT1 constitutively phosphorylated at the C-terminus. The latter possibility is also consistent with the fact that further addition of ATP could not increase binding to ITSN1SH3 A and pull down with the GST-fusion protein of VGLUT1 failed to precipitate ITSN1 (Voglmaier et al., 2006).

Generally, variations of extracellular glutamate appear to change diurnal behavior. The diurnally varying amounts of vesicular VGLUT are paralleled by reduced vesicular glutamate uptake (Yelamanchili et al., 2006). A reduction in vesicular glutamate concentration reduces release probability of glutamatergic SVs (Herman et al., 2014). In addition the glutamate transporter Eaat1 (excitatory amino acid transporter 1, also known as Glast and encoded by the gene Slc1a3) is subject to diurnal regulation and its expression is reduced in mice lacking a functional Per2 gene. The resulting hyper-glutamatergic state in Per2 mutant mice causes alterations at the behavioral level (Spanagel et al., 2005). The diurnal Enph/VGLUT1 interaction pattern resembles the expression of Eaat1, which is high at the beginning of the inactive light phase and low during the active dark phase (Spanagel et al., 2005). Eaat1 reduces extracellular glutamate and the here observed Enph/VGLUT1 interaction reduces release probability (Weston et al., 2011). The high amount of Enph/VGLUT1 interaction at CT12 under dark/dark conditions also appears to correlate with the high amount of VGLUT1 on SV at CT12 (Yelamanchili et al., 2006) unmasking a role of Enph circadian regulation of VGLUT1 endocytosis.
To conclude varying combinations of events decrease the amount of extracellular glutamate when animals are supposed to rest. In a day-night context different parameters, i.e., vesicular amounts of VGLUT1 dependent on the ITSN1SH3 A interaction and dynamin phosphorylation, Enph/VGLUT1 interaction, and Eaat expression may control extracellular glutamate and thereby allow specific fine tuning of glutamatergic synapses necessary for complex environmental adaption.

Acknowledgeent-The authors thank Antje Dräger, Birgit Metze and Marion Möbes for expert technical assistance. Work was supported by the Deutsche Forschungsgemeinschaft (SFB 958) A08).

\section{REFERENCES}

Aihara $\mathrm{Y}$, Mashima $\mathrm{H}$, Onda $\mathrm{H}$, Hisano $\mathrm{S}$, Kasuya $\mathrm{H}$, Hori T, Yamada S, Tomura H, Yamada Y, Inoue I, Kojima I, Takeda J (2000) Molecular cloning of a novel brain-type $\mathrm{Na}(+)$-dependent inorganic phosphate cotransporter. J Neurochem 74:2622-2625.

Albrecht U, Eichele G (2003) The mammalian circadian clock. Curr Opin Genet Dev 13:271-277.

Albrecht U, Foster RG (2002) Placing ocular mutants into a functional context: a chronobiological approach. Methods 28:465-477.

Armbruster M, Messa M, Ferguson SM, De Camilli P, Ryan TA (2013) Dynamin phosphorylation controls optimization of endocytosis for brief action potential bursts. eLife 2:e00845.

Bai J, Hu Z, Dittman JS, Pym EC, Kaplan JM (2010) Endophilin functions as a membrane-bending molecule and is delivered to endocytic zones by exocytosis. Cell 143:430-441.

Becher A, Drenckhahn A, Pahner I, Margittai M, Jahn R, AhnertHilger G (1999) The synaptophysin-synaptobrevin complex: a hallmark of synaptic vesicle maturation. J Neurosci 19:1922-1931.

Brunk I, Blex C, Rachakonda S, Holtje M, Winter S, Pahner I, Walther DJ, Ahnert-Hilger G (2006) The first luminal domain of vesicular monoamine transporters mediates G-protein-dependent regulation of transmitter uptake. J Biol Chem 281:33373-33385.

Daniels RW, Collins CA, Chen K, Gelfand MV, Featherstone DE, DiAntonio A (2006) A single vesicular glutamate transporter is sufficient to fill a synaptic vesicle. Neuron 49:11-16.

Darna M, Schmutz I, Richter K, Yelamanchili SV, Pendyala G, Holtje M, Albrecht U, Ahnert-Hilger G (2009) Time of day-dependent sorting of the vesicular glutamate transporter to the plasma membrane. J Biol Chem 284:4300-4307.

De Gois S, Jeanclos E, Morris M, Grewal S, Varoqui H, Erickson JD (2006) Identification of endophilins 1 and 3 as selective binding partners for VGLUT1 and their co-localization in neocortical glutamatergic synapses: implications for vesicular glutamate transporter trafficking and excitatory vesicle formation. Cell Mol Neurobiol 26:679-693.

Edwards RH (2007) The neurotransmitter cycle and quantal size. Neuron 55:835-858.

El Mestikawy S, Wallen-Mackenzie A, Fortin GM, Descarries L, Trudeau LE (2011) From glutamate co-release to vesicular synergy: vesicular glutamate transporters. Nat Rev Neurosci 12:204-216.

Evergren E, Gad H, Walther K, Sundborger A, Tomilin N, Shupliakov O (2007) Intersectin is a negative regulator of dynamin recruitment to the synaptic endocytic zone in the central synapse. J Neurosci 27:379-390.

Grabs D, Slepnev VI, Songyang Z, David C, Lynch M, Cantley LC, De Camilli P (1997) The SH3 domain of amphiphysin binds the proline-rich domain of dynamin at a single site that defines a new SH3 binding consensus sequence. J Biol Chem 272:13419-13425. 
Gronborg M, Pavlos NJ, Brunk I, Chua JJ, Munster-Wandowski A, Riedel D, Ahnert-Hilger G, Urlaub H, Jahn R (2010) Quantitative comparison of glutamatergic and GABAergic synaptic vesicles unveils selectivity for few proteins including MAL2, a novel synaptic vesicle protein. J Neurosci 30:2-12.

Herman MA, Ackermann F, Trimbuch T, Rosenmund C (2014) Vesicular glutamate transporter expression level affects synaptic vesicle release probability at hippocampal synapses in culture. $\mathrm{J}$ Neurosci 34:11781-11791.

Kohansal-Nodehi M, Chua JJ, Urlaub H, Jahn R, Czernik D (2016) Analysis of protein phosphorylation in nerve terminal reveals extensive changes in active zone proteins upon exocytosis. eLife 5.

Milosevic I, Giovedi S, Lou X, Raimondi A, Collesi C, Shen H, Paradise S, O'Toole E, Ferguson S, Cremona O, De Camilli P (2011) Recruitment of endophilin to clathrin-coated pit necks is required for efficient vesicle uncoating after fission. Neuron 72:587-601.

Moechars D, Weston MC, Leo S, Callaerts-Vegh Z, Goris I, Daneels G, Buist A, Cik M, van der Spek P, Kass S, Meert T, D'Hooge R, Rosenmund C, Hampson RM (2006) Vesicular glutamate transporter VGLUT2 expression levels control quantal size and neuropathic pain. J Neurosci 26:12055-12066.

Ni B, Rosteck Jr PR, Nadi NS, Paul SM (1994) Cloning and expression of a cDNA encoding a brain-specific $\mathrm{Na}(+)$ dependent inorganic phosphate cotransporter. Proc Natl Acad Sci U S A 91:5607-5611.

Pechstein A, Bacetic J, Vahedi-Faridi A, Gromova K, Sundborger A Tomlin N, Krainer G, Vorontsova O, Schafer JG, Owe SG, Cousin MA, Saenger W, Shupliakov O, Haucke V (2010) Regulation of synaptic vesicle recycling by complex formation between intersectin 1 and the clathrin adaptor complex AP2. Proc Natl Acad Sci U S A 107:4206-4211.

Pechstein A, Gerth F, Milosevic I, Japel M, Eichhorn-Grunig M, Vorontsova O, Bacetic J, Maritzen T, Shupliakov O, Freund C, Haucke V (2015) Vesicle uncoating regulated by $\mathrm{SH} 3-\mathrm{SH} 3$ domain-mediated complex formation between endophilin and intersectin at synapses. EMBO Rep 16:232-239.

Ringstad N, Nemoto Y, De Camilli P (2001) Differential expression of endophilin 1 and 2 dimers at central nervous system synapses. J Biol Chem 276:40424-40430.

Santos MS, Foss SM, Park CK, Voglmaier SM (2014) Protein interactions of the vesicular glutamate transporter VGLUT1. PLoS One 9:e109824.
Spanagel R, Pendyala G, Abarca C, Zghoul T, Sanchis-Segura C Magnone MC, Lascorz J, Depner M, Holzberg D, Soyka M, Schreiber S, Matsuda F, Lathrop M, Schumann G, Albrecht U (2005) The clock gene Per2 influences the glutamatergic system and modulates alcohol consumption. Nat Med 11:35-42.

Takamori S, Holt M, Stenius K, Lemke EA, Gronborg M, Riedel D, Urlaub H, Schenck S, Brugger B, Ringler P, Muller SA, Rammner B, Grater F, Hub JS, De Groot BL, Mieskes G, Moriyama Y, Klingauf J, Grubmuller H, Heuser J, Wieland F, Jahn R (2006) Molecular anatomy of a trafficking organelle. Cell 127:831-846.

Tsanov M, Manahan-Vaughan D (2007) The adult visual cortex expresses dynamic synaptic plasticity that is driven by the light/dark cycle. J Neurosci 27:8414-8421.

Vinatier J, Herzog E, Plamont MA, Wojcik SM, Schmidt A, Brose N, Daviet L, El Mestikawy S, Giros B (2006) Interaction between the vesicular glutamate transporter type 1 and endophilin A1, a protein essential for endocytosis. J Neurochem 97:1111-1125.

Voglmaier SM, Edwards RH (2007) Do different endocytic pathways make different synaptic vesicles? Curr Opin Neurobiol 17:374-380.

Voglmaier SM, Kam K, Yang H, Fortin DL, Hua Z, Nicoll RA, Edwards $\mathrm{RH}$ (2006) Distinct endocytic pathways control the rate and extent of synaptic vesicle protein recycling. Neuron 51:71-84.

Weston MC, Nehring RB, Wojcik SM, Rosenmund C (2011) Interplay between VGLUT isoforms and endophilin $\mathrm{A} 1$ regulates neurotransmitter release and short-term plasticity. Neuron 69:1147-1159.

Yelamanchili SV, Pendyala G, Brunk I, Darna M, Albrecht U, AhnertHilger G (2006) Differential sorting of the vesicular glutamate transporter 1 into a defined vesicular pool is regulated by light signaling involving the clock gene Period2. J Biol Chem 281:15671-15679.

Zander JF, Munster-Wandowski A, Brunk I, Pahner I, Gomez-Lira G, Heinemann U, Gutierrez R, Laube G, Ahnert-Hilger G (2010) Synaptic and vesicular coexistence of VGLUT and VGAT in selected excitatory and inhibitory synapses. J Neurosci 30:7634-7645.

Zheng B, Larkin DW, Albrecht U, Sun ZS, Sage M, Eichele G, Lee CC, Bradley A (1999) The mPer2 gene encodes a functional component of the mammalian circadian clock. Nature 400:169-173. 